\title{
APPROACH FOR GENERATION OF COMPLETE VIRTUAL MACHINE TOOLS BASED ON A UNIFIED MODEL DESCRIPTION
}

\author{
ERLER, M[artin] \& ESSERS, M[arius]
}

\begin{abstract}
Generation of controllable machine tool models is essential for virtualization of manufacturing processes. Though the generation is still very time-consuming, main applications will be found in expensive software with proprietary model descriptions. The presented approach allows the generation of complete machine tool models in a really short time. The generated models are fully controllable and also combine with an interface suitable to establish a connection to any $\mathrm{CNC}$ control.This is feasible by unifying the description for the model's kinematics and its graphics attributes. Based on the unified model description, a fully controllable machine tool model can be generated automatically using forward transformation and linking the graphics data to it. It is also possible to add physical aspects and behavior to the machine model and its components. Through the consequent description as modular parts, the axes of the machine tool become interchangeable.First, virtual machine tools are successfully created by applying the integrated module for visualization. These machines can be screened as a part of a single application and can be scaled up to fully immersive environments.
\end{abstract}

Keywords:Virtual Machining, Virtual Manufacturing, Virtual Reality, Virtual Machine Tooling, Virtual Machine

\section{METHODOLOGICAL CONCEPT ${ }^{1}$}

The use of Virtual Reality (VR) in the industry is becoming increasingly important. It represents an ideal medium for interdisciplinary collaboration, if it is involved at an early stage in development and production. The specific usestrongly accelerates the product and production development processes. Main fields of application are in simultaneous engineering as well as for a holistic view of processes based on the product, the production, and the utilized resources.VR has great potential for an iterative approach, as it is common within product and process planning and design. In production technology, VR can be used for spatial visualization, multimedia information representation, storage, as well as interactive simulation[01]. Therefore, VR-aided NC-based planning, programming, and editing has become state of the art. In every stage of a product development process or production planning process different techniques and technologies are used.

The creation of NC programs is done with support of VR, although only in use of a workstation monitor[02], [03], [04], [05]. These NC-programs can be processed further by simulation and validation regarding the

${ }^{1}$ With the kind support of: ZentrumVirtuellerMaschinenbau TU Dresden, OPS-INGERSOLL Funkenerosion GmbH and andron $\mathrm{GmbH}$ contained machining parameters and tool paths to perform minor adjustments for optimizations [06], [07]. Coupled with a real numerical controller, the simulation of a NC-program gets much closer to the shop floor. In use of this coupling with a hardware controller[08], [09], and a software controller [10],some commercial and noncommercial systems are known.

Besides the use of a simple monitor of a workstation for 3D-modeling and visualization, the benefits of VR can be extended. This will be achieved in two ways. First, by the use of screening technologies with a higher grade of immersion, as given by monitors or projectors with stereoscopic functionalities. Beyond this, there is a much more elaborate screening technology realized in a Cave Automatic Virtual Environment (CAVE) [11], [12], [13]. Objects can be screened in their natural size and with a stereoscopic effect as well. Therefore, it is possible to reach a very high degree of immersion.

The second way to extend the use of VR is to factor in the physical attributes of machine tools and machining. By calculating process forces and torques, machining parameters can be controlled and simulated, regarding their results in productivity [14] and work piece quality [15], [16]. This effort is done to reduce production time and costs, and to increase the production quality. These main goals of production are just some reasons for the simulation of machines and machining. In most cases, the kinetic machine characteristics are forming the base of simulation, regardless of whether they are used for simple visualization or for advanced virtualization. Due to the large number of different machine tools and even the larger number of the possible machine configurations, fast and automated ways to build virtual equivalents are needed.

\section{RAPID CREATION OF VIRTUAL MACHINES}

The method presented for the efficient and rapid development of a virtual machine expands the possible application scenarios significantly. At the same time, a much better reflection of details in the environment becomes feasable by spatial localization. The method developed allows the use of these benefits at an economically viable large scale and sets the basis for further virtualization methods.

\subsection{Requirements}

In order to use the virtual machine as a software tool, it must be adapted quickly to its specific task. In the fields of research and development, common 


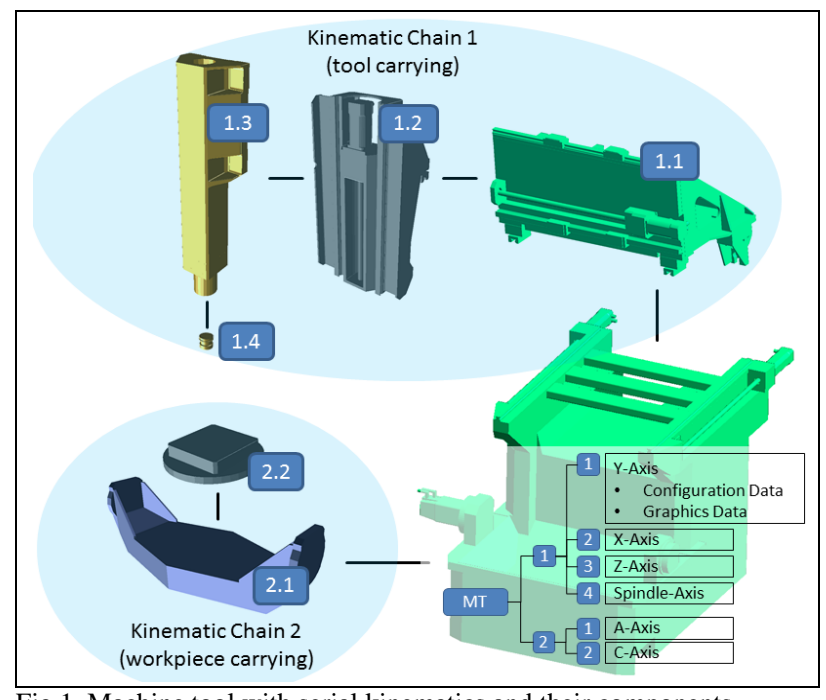

Fig.1. Machine tool with serial kinematics and their components

configuration changes as part of trials and tests are normal. For education purposes, a wide range of different machines and configurations is attractice to support the curriculum. For the transfer of the models in SME, company-specific design variants are a point of interest.

Machine tools typically are based on a serial kinematics, which is modeled and linked to represent suitable graphics for the need to obtain a controllable virtual machine. The main work in the construction of such a model is the development of the kinematics based on the machine configuration and the linkage of the graphic data with each axis (Fig. 1). The underlying mechanism of transformation of the original CAD data to graphic data and its enrichment with machine functions (Fig. 2) could be largely generalized.

\subsection{Generalized Virtual Machine Design}

To achieve this, the links are not placed in the foreground kinematic reprasentation, but rather the axes, which correspond to the real components. This replica is more realistic and has the consequence that the position of the axes to one another is adjustable. Also,there is no need for additional parameters such as in the $\mathrm{DH}$ convention (Denavit and Hartenberg) [17].

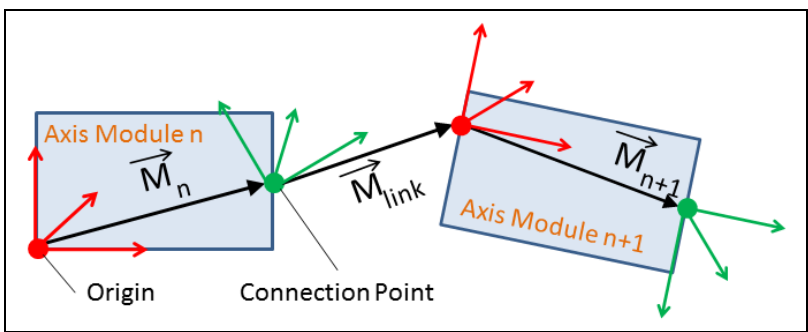

Fig.3. Transformation description for two axis modules

The mobility of the modeland thus anaxis chainis achieved by the decomposition of an axis intotwo axis modules whosetranslation orrotation to each other occursover the link function.

For this purpose, an axis module is defined through an origin and a connection point, their position and orientation to one another are described through a transformation matrix using homogeneous coordinates.

Additionally, the link is finally realized through atransformation matrix that describes the transformation from one connection point to the origin of the following axis module (Fig. 3). There are three distinct module types:

1. the axis chain (contains one or more axes),

2. the axis (contains two axis modules)

3 . and the axis module.

By using more than one axis chain, an arbitrary machine tool movement can be performed by automatically providing a fully movable serial kinematics.

The entirely parameterized interface and the structure of the machine tool axis-configuration and visualization data is stored. Through this unified description method, a controllable machine model can be generated automatically for any machine tool with serial kinematics. The semi-automated generation of the graphics data from the CAD data and their linkage with the controllable machine model reduces the time needed to build a virtual machine again. The significant total time saving approached during the process of virtualization enables a wide range of applications.

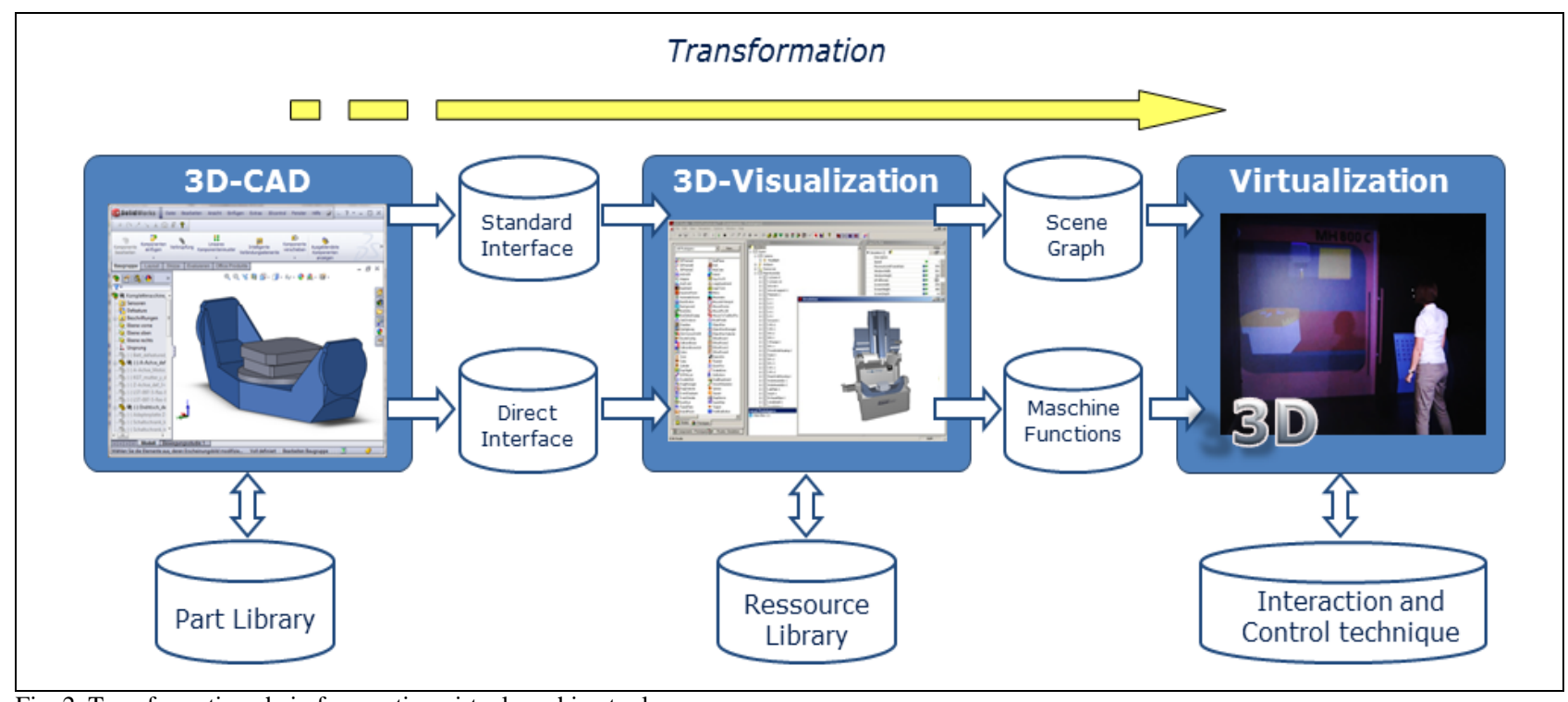

Fig. 2. Transformation chain for creating virtual machine tools 


\section{OPERATION, CONTROL, AND CONFIGURATION OF VIRTUAL MACHINES AND THEIR COMPONENTS}

Virtualization is used, among other things, mainly for illustration andoffers an optimumamount of adaptation options for displaying. The choice between different predefined views is just as possible as the free movement. This happens in an intuitive way, either via a 3D mouse, a flystick, a coupled tracking system, or by usinga simple set of mouse and keyboard. This wide variety of tethered peripherals offers a wide choice of input devices for several environments. Moreover, the selection and change of views through an external numerical controller or PLC is possible. This allows complex view scenarios adjusted to a specific machining situation. Possible are camera movements (e.g. along the tool path to the magazine or the tool change) or fade in and focusing onindividual machine components (Fig.4). The combination of these techniques enables the user the ability to take perspectives and generate views that are not possible in reality. This leads toan opportunity to indicate effects between the process, machine tool, and machining much better and faster.

The possibilities of the virtual machine can be utilized to investigate the behavior of certain components or machinery. Therefore, a direct control interfacewas developed which allows the triggering and operation of central functions (e.g. axis movements or opening of the work area enclosure). Here, again, the aforementioned input devices are usable. The described functionalities are fully integrated in the automatic mode as well. By connecting the virtual machines to a numerical control and / or PLC, the entire production process, including the connected peripherals, isvisualized. The NC-program flow is either via the coupling with a real numerical controller / PLC (Hardware in the Loop), or via the connection with a virtual numerical controller / PLC (software in the Loop). Thus, any common program can be interpreted. Likewise, the connection of an internal $\mathrm{NC}$-interpreter is possible. The virtual machine represents a comprehensive

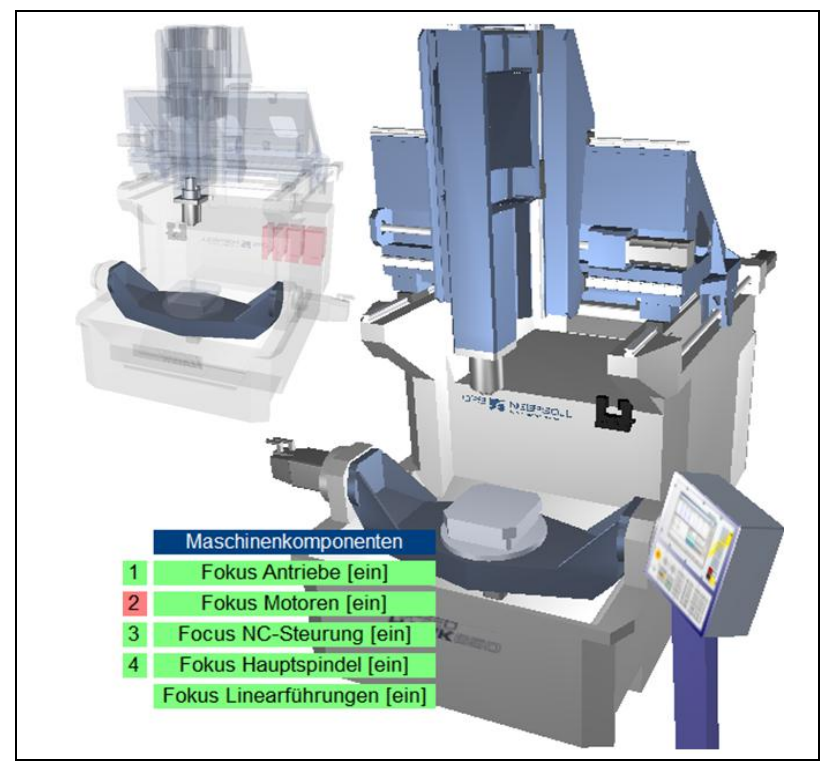

Fig. 4. Virtual machine tool OPS Ingersoll SH 650 with user interface (r.) and transparent machine parts (1.)



Fig.5.Control alternatives for virtual machines

simulation tool referring to the possibility of different ways to receive tool paths (Fig. 5).

Since interaction in simulations is increasingly becoming important, the virtual machine was equipped with a bidirectional communication interface. Thisinterface handles responses of virtual machinespecific functions (such as tool change or probing), but also its peripheral components (e.g. surveying tool) in feedback with the simulated process. This means that the machine function,e.g.a tool measuring process, can be fully simulated - including the return of the measured data. Even in handling any collisions that are detected within the virtual machine can be sent to the controller and causes an emergency stop.Through the consistent modular design of the virtual components, further data can be exchanged as well. Thus, the transmission of tool data basics (length, diameter, radius corner) is already implemented.

\section{APPLICATION SCENARIOS}

The use of virtual machines brings benefits to machine tool manufacturers and control manufacturers, as well as to their users. Machine tool manufacturers are able to perform functional tests of adjustable and controllable machines and their components without the need to have a physical system. Withan easy replacement within a short time, a variety of machine configurations can be tested. The created virtual configurations can be utilized for training in order to identify problems before the actual machine installation and helps avoid them afterwards.

As a second group manufacturers of NC-controllers can benefit from virtual prototypes for different machine configurations, since the controller can already be adapted while the real machine is under construction, or even in the planning stage. If physical properties are integrated in the virtual machine which is used on a numerical control, look-ahead functionality can be improved by avoiding critical situations in the process.

The third group represents users with the areas of education and training. Significant cost reductions can be approached by the use of virtual machines within the NC-training (Fig. 6):

1. No valuable machining capacity is used

2. Manual inputs processed by the numerical control can be comprehended without the risk of damaging any equipment

3. The spatial and space required is much lower. 


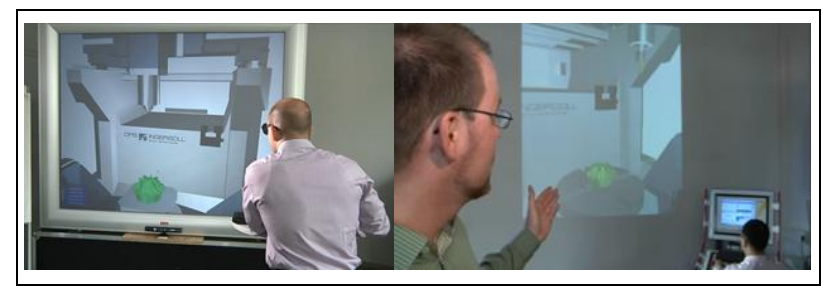

Fig. 6.Use of a stereoscopic projection wall and a NC-controller

A high level of detail provides access to the field of numerical control planning. Production data is used for code validation, verification of process safety (Fig. 7), and suitability of the process components, e.g. clamping. The expansion of the use of virtual manufacturing centered in the production planning will be the subject of research in the next few years.

\section{FUTURE PROSPECTS}

For further research, two goals have been identified. On the one hand, real-time models for material removal with a high spatial resolution need to be implemented into machine models for a proper use in validation of NC-programs, prototypes of machines, and their components, as well as the coupling with an NCcontroller. On the other hand, efforts need to bedone to extend the physical properties of the manufacturing systems as a whole. Especially the machine, workpiece, and tool rigidities are essential for a dynamic consideration of the cutting process. This means chatter or vibration effects and the dynamic change of cutting condition occurring, caused by deformations of the system. The influence of the kinetic behavior will be taken into consideration as well.

Another topic is the modeling of mechatronic components and the analysis of their influence on the cutting process, as well as the energy consumption while cutting. The results of this can be used to optimize the energy efficiency.

Next to an extensive use of virtual machines in the product planning and design phase, which supports the planner / designer with an intensive, comprehensive, and effective overview of his work item, the holistic approach of the machining system provides the opportunity to use and take into account all characteristics of the system while production planning is being done. A prototypic system for which provides the possibility to plan processes with an optimum amount of productivity will be developed in the next two years.

\section{CONCLUSION}

The method presented for the efficient and rapid development of a virtual machine expands the possible application scenarios significantly. Due to the lower costs,resulting in a shorter time and lower exerted effort to develop a virtual machine, the use of a virtual machine is also becomingeconomically reasonable for training and product presentation purposes. Above all, virtual machines can be widely used for engineering and design. At the same time, a much better reflection of details in the environment becomes feasible by spatial localization. The method developed allows the use of these benefits at an economically viable large scale and sets the basis for further virtualization methods.
The modular design of the virtual machine supports the exchange and extensibility of components to each other, which further increases the usability value. Also,with the implementation as an open-system simulation software, the virtual machine may be integrated with moderate effort or it can be equipped with another virtual machine.

\section{REFERENCES}

[1] Bilalis, N. ; Petousis, M. ; Antoniadis, A.: Model for surfaceroughness parameters determination in a virtual machine shop environment. In: The International Journal of Advanced Manufacturing Technology 40 (2009), 11-12, S. 1137-1147

[2] Parametric Technology Corporation: Creo Parametric. URL: http://creo.ptc.com,Accessed: 2012-07-13

[3] Dassault Systèmes: DELMIA. URL: http://www.3ds.com., Accessed: 2012-07-13

[4] EXAPT Systemtechnik GmbH, URL: http://www.exapt.de,Accessed: 2012-07-13

[5] Frankel, Aaron: Machining Line Planner, URL: http://www.plm.automation.siemens.com/en_us/Images/16796_tc m1023-82692.pdf, Accessed: 2012-07-13

[6] Siemens AG: Tecnomatix em-RealNC,URL: http://www.plm.automation.siemens.com/de_de/products/tecnom atix/index.shtml,Accessed: 2012-07-12

[7] CGTech Deutschland GmbH: VERICUT. URLhttp://www.cgtech.com, Accessed: 2012-07-13

[8] Kao, Y. C. ; Cheng, H. Y. ; Chen, Y. C.: Development of a virtual controller integrating virtual and physical CNC. In: Progress On Advanced Manufacture For Micro/Nano Technology 2005, PT 1 AND 2 505-507 (2006), S. 631-636

[9] Bosch Rexroth AG: IndraMotion MTX. URL http://www.boschrexroth.com/business_units/brc/de/branchen_d e/sim_de/sim_sys_de_neu/MTX_compact_de/index.jsp Accessed: $2012-07-13$

[10] Frankel, Aaron: NX CAM - Siemens Sinumerik Virtual NC Controller Kernel (2011)

[11] Department of mechanical Engineering: Virtual Reality Center Production Engineering. URL: https://www.tuchemnitz.de/mb/WerkzMasch/vrcp/outline_vpe.php,Accessed: 2012-07-13

[12] Fraunhofer-Gesellschaft: Virtual Development, Engineering and Training VIDET, Magdeburg

URL:http://www.fraunhofer.de/en/institutes-researchestablishments/innovation-clusters/virtual-developmentengineering-training.html, Accessed: 2012-07-13

[13] Dresden University of Technology: Zentrum Virtueller Maschinenbau. URL: http://tu-dresden.de/mw/zvm,Accessed 2012-07-13

[14] Third Wave Systems: Production Module. URL: http://www.thirdwavesys.com/, Accessed: 2011-12-10

[15] Lee, Seok Won ; Nestler, Andreas: Tool load balancing at simultaneous five-axis ball-end milling via the NC simulation kernel : 1st CIRP Conference on Virtual Machining and Production Technology (CIRP VMPT) (2012), Paper 33, 28th May -1st June 2012, Montréal, Canada

[16] Lee, Seok Won ; Nestler, Andreas: Complete swept volume generation, Part I: Swept volume of a piecewise C-1-continuous cutter at five-axis milling via Gauss map. In: COMPUTERAIDED DESIGN 43 (2011), Nr. 4, S. 427-441

[17] Denavit, J. ; Hartenberg, R.: A kinematic notation for lower-pair mechanisms based on matrices. In: Journal Of Applied Mechanics, 1955, S. 215-221 